\title{
CORRIGENDUM
}

\section{Magic ARB, or magic trial?}

\section{Iwao Kuwajima}

Hypertension Research (2010) 33, 755; doi:10.1038/hr.2010.87

Correction to: Hypertension Research (2010) 33, 414; doi:10.1038/ hr.2010.34

The authors of the above article noted an error in publication of this paper (AOP and in the May 2009 issue) in the affiliation address. The correct address is shown below.

Dr I Kuwajima is at the Division of Cardiology, Tokyo Metropolitan Geriatric Hospital, 35-2 Sakaecho, Itabashi-ku, Tokyo 173-0015, Japan.

E-mail: kuwajima@tmghig.jp 